\title{
Heartbeat: Improving diagnosis and management of aortic
} valve disease

Many adults with severe aortic stenosis (AS) present with end-stage disease, at a point where intervention is risky and might be futile, because the diagnosis is missed earlier in the disease course. Given the increasing prevalence of AS in our ageing populations and the effectiveness of transcatheter valve implantation for treatment of this highly mortal disease, accurate early diagnosis is increasingly important. Physicians and patients have faith that the stethoscope is an adequate approach to diagnosis of valve disease; indeed, the stethoscope is the symbol of a doctor. Yet, numerous studies have shown that cardiac auscultation is inaccurate for diagnosing the presence or severity of valve disease, regardless of training and experience. We find it difficult to acknowledge that the sounds generated by the heart, no matter how carefully listened to or recorded, simply are not a reliable reflection of the presence or severity of valve disease.

In this issue of Heart, Gardezi and colleagues $^{1}$ evaluated the accuracy of cardiac auscultation by experienced general practitioners in 251 adults over age 65 years undergoing echocardiography. Overall, $68 \%$ of patients had a new diagnosis of mild valvular heart disease (VHD) and 14\% had significant VHD as defined by echocardiographic findings. The sensitivity of cardiac auscultation was very low, even for significant VHD (44\%) with likelihood ratios that were not statistically significant (table 1 ).

Why are physicians so reluctant to admit that cardiac auscultation is no longer an adequate approach to diagnosis of valve disease? Certainly, we still should listen to our patients-both with a careful medical history and with our stethoscopes-because auscultation remains useful for many other conditions, such as heart failure. In addition, the thrill of hearing a murmur and deducing the underlying lesion will remain a rite of transition for all medical trainees. However, perhaps it is time to consider screening older adults with echocardiography for

Correspondence to Prof. Catherine M Otto, Division of Cardiology, University of Washington, Seattle, Washington 98195, USA; cmotto@uw.edu, cmotto@u. washington.edu
Table 1 Accuracy of cardiac auscultation by general practitioners and cardiologists in the diagnosis of VHD, compared with echocardiography as the reference standard $(95 \% \mathrm{Cls}$ in parentheses)

Significant VHD

(A) General practitioners

\begin{tabular}{lllrl|} 
& Positive & Negative & Total & \\
\hline Murmur present & 16 & 66 & 82 & PPV: $20 \%(12 \%$ to $30 \%)$ \\
\hline Murmur absent & 20 & 149 & 169 & NPV: $88 \%(82 \%$ to $93 \%)$ \\
\hline Total & 36 & 215 & 251 & Prev: $14 \%(10 \%$ to $19 \%)$ \\
& Sens: $44 \%(28 \%$ to $62 \%)$ & Spec: $69 \%(63 \%$ to $75 \%)$ & & \\
& LR+: $1.45(0.95$ to 2.20$)$ & LR-: $0.80(0.59$ to 1.09$)$ & &
\end{tabular}

(B) Cardiologists (mean rating)

\begin{tabular}{lllll} 
& Positive & Negative & Total & \\
\hline Murmur present & 11 & 40 & 51 & PPV: $22 \%$ (11\% to $35 \%)$ \\
Murmur absent & 25 & 175 & 200 & NPV: $88 \%$ (82\% to $92 \%)$ \\
\hline Total & 36 & 215 & 251 & Prev: $14 \%$ (10\% to 19\%) \\
\hline & Sens: $31 \%(16 \%$ to $48 \%)$ & Spec: $81 \%(76 \%$ to $86 \%)$ & & \\
\hline & LR+: $1.64(0.93$ to 2.89$)$ & LR-: $0.85(0.68$ to 1.07$)$ & & \\
\hline
\end{tabular}

VHD, valvular heart disease., PPV, positive predictive value; NPV, negative predictive value; Sens sensitivity; Sepc, specificity; LR, likelihood ratio; Prev, prevalance
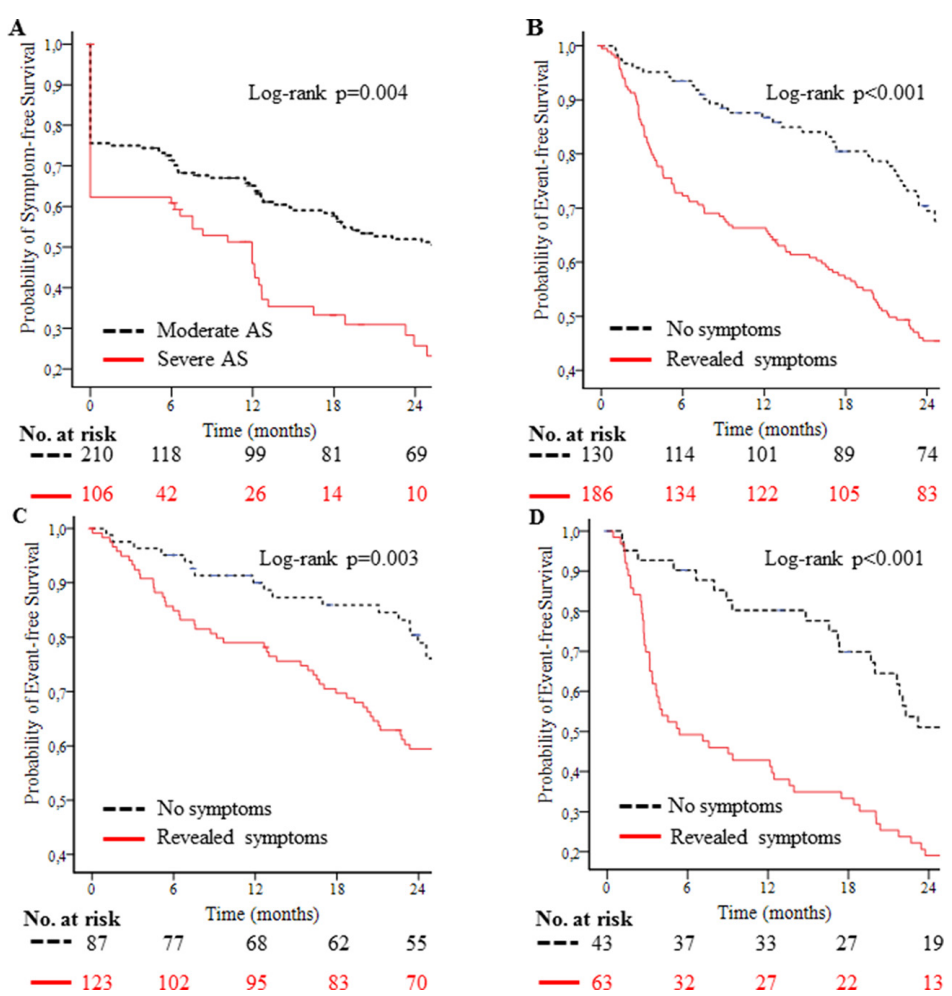

Figure 1 Kaplan-Meier plots showing the probability of symptom-free survival according to the severity of AS (A) and event-free survival according to symptoms during ETT in entire study population (B), moderate AS (C) and severe AS (D). AS, aortic stenosis; ETT, exercise treadmill test. 


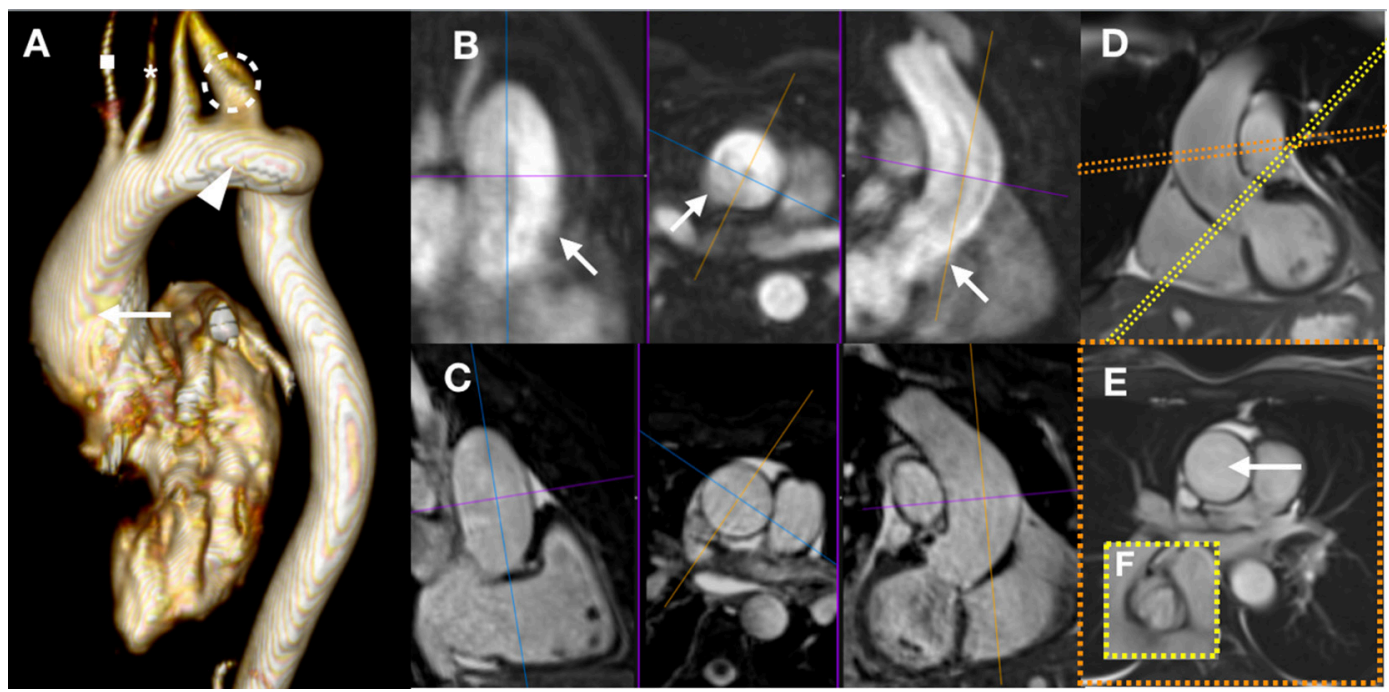

Figure 2 Evaluation of aortic dissection risk by imaging in Turner syndrome (TS) includes non-invasive evaluation of the aortic valve, aortic arch phenotype and thoracic aortic diameter. A combination of transthoracic echocardiography and cardiovascular magnetic resonance (CMR) is often needed with cardiovascular CT performed in select girls and women. The addition of CMR is increasingly needed with age, as acoustic windows often become limited and the entire thoracic aorta must be assessed. Accurate aortic size measurements require ECG-gated imaging, especially for the ascending aorta, and three-dimensional (3D) data with multiplanar reconstructions or dedicated two-dimensional (2D) 'double-oblique' imaging in order to optimise measurement variability. Here, different CMR sequences are shown for a 35-year-old woman with TS under consideration for prophylactic aortic surgery. A 3D volume-rendered reconstruction of a contrast-enhanced, non-ECG-gated aortogram (A) shows: (1) a left-sided aortic arch with incidental separate arch origins of the right common carotid ( $A$, asterisk) and right subclavian ( $A$, square) arteries, $(2)$ a dilated origin of the left subclavian artery ( $A$, circle) with less dilated origin of the preceding left common carotid artery, (3) an elongated and mildly tortuous but unobstructed aortic arch ( $A$, arrowhead) and (4) the point of maximum dilation in the mid-ascending aorta ( $A$, arrow). The same 3D data set allows multiplanar reconstructions for orthogonal diameter measurements at any point (B). However, the non-ECG-gated nature of the aortogram causes blurring of the aortic wall from cardiac motion leading to inaccurate measurement ( $B$, arrows); this is less pronounced for the descending aorta. Data acquisition for a particular cardiac phase minimises any such blurring as seen in a multiplanar reformat of a non-contrast-enhanced ECG-triggered 3D volumetric data set (C). A specific cardiac phase can also be assessed with 2D imaging as is shown for an ECG-gated cine that is carefully positioned for the correct image plane ( $D$, orange slice position) giving an orthogonal plane through the point of maximum aortic diameter (E with arrow indicating the mid-ascending aorta). The same principle is used for en face visualisation of the bicuspid aortic valve ( $D$, yellow slice position giving the orthogonal plane (F)). The peak-systolic mid-ascending aortic diameter was $2.6 \mathrm{~cm} / \mathrm{m}^{2}$, and the recommendation was for prophylactic aortic surgery from the multidisciplinary team at a specialist centre for adults with congenital heart disease.

VHD, especially given the increasing availability of low-cost point-of-care ultrasound (POCUS) devices. My view is that "Now is the time to start teaching POCUS to healthcare providers. The diagnosis of heart valve disease is missed in too many patients. We need to 'mind the gap' by putting our stethoscopes back in our pockets and taking out our ultrasound transducers." 2

Another vexing issue in adults with AS is recognising symptom onset. Many older adults have symptoms of decreased exercise tolerance, dyspnoea, dizziness or chest pain that may or may not be related to aortic valve obstruction. In addition, because symptom onset is insidious, patients often do not recognise a gradual limitation as a 'symptom' and thus do not report these changes to their physician. In a retrospective cohort study of 316 older adults with apparently asymptomatic moderate to severe AS, Saeed and colleagues ${ }^{3}$ found that $29 \%$ had symptoms on exercise testing at baseline. Their outcome data confirm that symptoms provoked by exercise testing indicate the patient is symptomatic with a 24-month event-free survival of only $46 \% \pm 4 \%$ in those with symptoms on stress testing compared with $70 \% \pm 4 \%$ in those who remained asymptomatic $(\mathrm{p}<0.0001)$ (figure 1).

Bicuspid aortic valve disease is present in 15\%-30\% of women and girls with Turner syndrome (TS). In addition, TS is associated with a risk of aortic dissection 20-100 times higher than women without TS (figure 2). Mortensen and colleagues ${ }^{4}$ provide an excellent review article on cardiovascular imaging in patients with TS, detailing recommendations for screening and follow-up of these highrisk patients. Periodic imaging is recommended in women and girls with TS, even in the absence of other risk factors and even when baseline aortic size appears to be normal (figure 3).

The Education in Heart article ${ }^{5}$ in this issue discusses the principles of managing patients with VHD who are undergoing non-cardiac surgery. Management starts with an accurate diagnosis of the severity of VHD, usually by echocardiography, along with assessment of surgical risk. During the procedure, invasive haemodynamic monitoring and optimisation of loading conditions by an experienced anaesthetist is essential with addition specific recommendation for each type of valve lesion. Postoperatively, continued close monitoring with rapid intervention for hypotension, electrolyte imbalances or arrhythmias is recommended.

You can then test your knowledge with the Image Challenge case ${ }^{6}$ which presents an 82-year-old man with a systolic murmur undergoing preoperative evaluation for non-cardiac surgery. The Doppler tracings show an interesting finding that impacted patient care.

\section{Competing interests None declared.}

Provenance and peer review Commissioned; internally peer reviewed.

(c) Author(s) (or their employer(s)) 2018. No commercial re-use. See rights and permissions. Published by BMJ.

Check for updates 


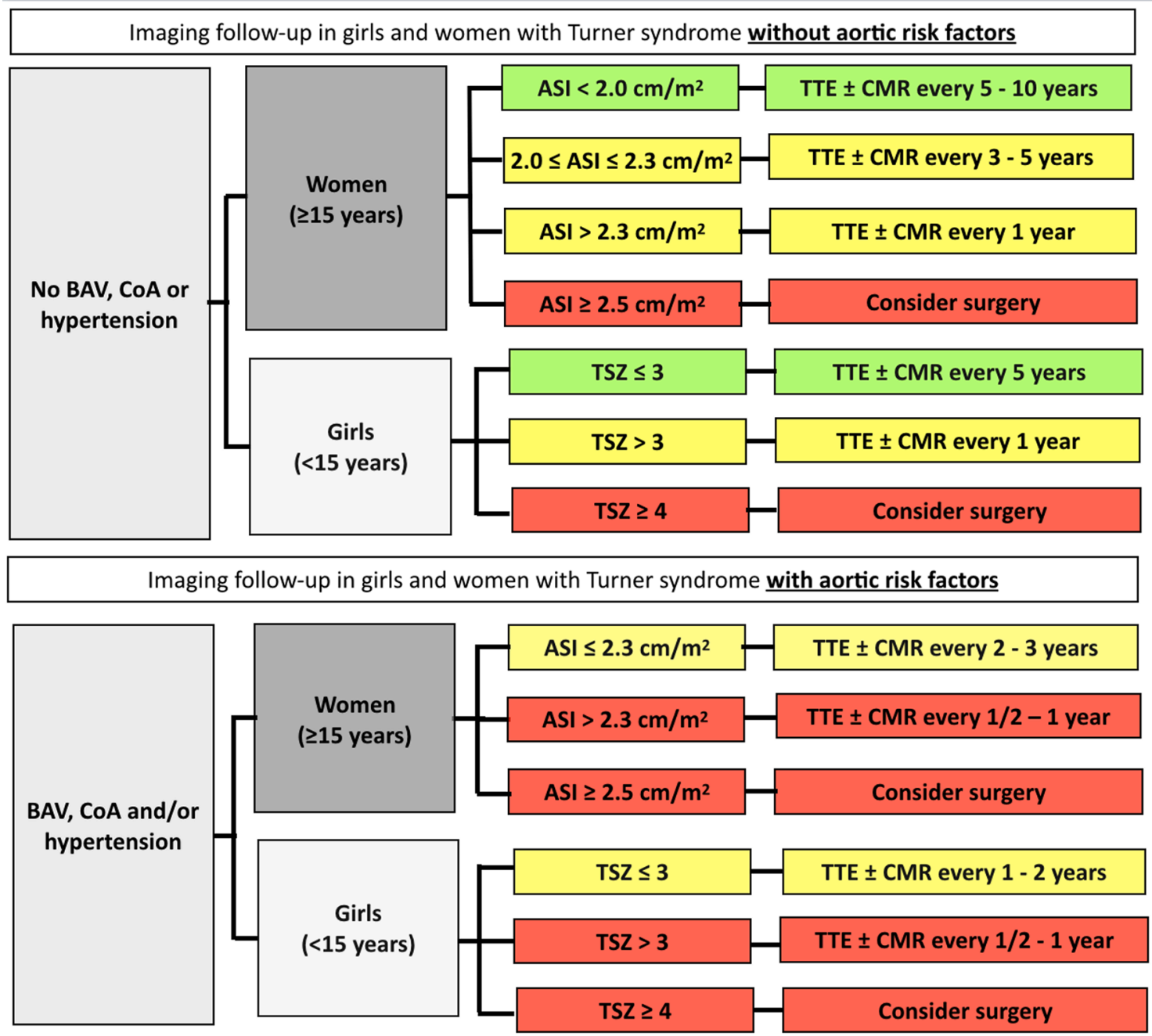

Figure 3 Imaging follow-up for aortic dilation in girls and women with Turner syndrome (TS) should be adapted to age, aortic size and aortic risk factors as per expert consensus in the international guidelines Risk factors include bicuspid aortic valve (BAV), aortic coarctation (CoA) and hypertension as diagnosed at the initial cardiovascular screening. This initial screening includes transthoracic echocardiography (TTE), cardiovascular magnetic resonance (CMR) and ambulatory blood pressure measurement. The aortic size criteria apply to the mid-ascending aorta at the level of the right pulmonary artery, and this size is given as the aortic diameter indexed to body surface area (ASI) in adult women and TS-specific z-scores (TSZ) in girls. No evidence exists to guide similar thresholds for other aortic segments in TS. The colours signify perceived risk: green is low, yellow is moderate and red is high. The addition of CMR to TTE should be determined by the quality of the acoustic windows, and remembering that TTE is less accurate for aortic disease that extends beyond the aortic sinuses when compared with CMR. CMR is more accurate than TTE owing to this modality being independent of standard imaging planes. If CMR is contraindicated or not feasible, then cardiovascular CT may be used as an alternative, but the associated radiation exposure should be considered.

To cite Otto CM. Heart 2018;104:1807-1809.

Heart 2018;104:1807-1809.

doi:10.1136/heartjin-2018-314232

\section{REFERENCES}

1 Gardezi SKM, Myerson SG, Chambers J, et al. Cardiac auscultation poorly predicts the presenceof valvular heart disease in asymptomatic primarycare patients. Heart 2018;104:1832-5.

2 Otto CM. Mind the gap: missed valve disease diagnosis. Heart 2018;104:1810-1.

3 Saeed S, Rajani R, Seifert R, et al. Exercise testing in patients with asymptomatic moderate or severe aortic stenosis. Heart 2018;104:1836-42.

4 Mortensen KH, Young L, De Backer J, et al. Cardiovascular imaging in Turner syndrome: state-of-the-art practice across the lifespan. Heart 2018;104:1823-31.

5 Chambers JB. Valve disease and non-cardiac surgery. Heart 2018;104:1878-87.

6 Kupsky DF, Ananthasubramaniam K, Lee J. Eightytwo-year-old man with a systolic murmur. Heart 2018;104:1887. 